WIS-94/3/Jan-PH

January 1994

\title{
Phenomenology of models with more than two Higgs doublets
}

\author{
Yuval Grossman \\ Department of Particles Physics \\ The Weizmann Institute of Science \\ Rehovot 76100, ISRAEL
}

\begin{abstract}
We study the most general Multi-Higgs-Doublet Model (MHDM) with Natural Flavor Conservation (NFC). The couplings of a charged scalar $H_{i}^{ \pm}$to up quarks, down quarks and charged leptons depend on three new complex parameters, $X_{i}, Y_{i}$ and $Z_{i}$, respectively. We prove relations among these parameters. We carry out a comprehensive analysis of phenomenological constraints on the couplings of the lightest charged scalar: $X, Y$ and $Z$. We find that the general MHDM may differ significantly from its minimal version, the Two-Higgs-Doublet Model (2HDM).
\end{abstract}




\section{Introduction}

The Higgs sector of the Standard Model (SM), consisting of a single Higgs doublet, has not yet been experimentally tested. The possibility of an extended Higgs sector is definitely consistent with experimental data. The simplest extensions are models with several Higgs doublets, implying the existence of charged scalars. The simplest of these is the Two-Higgs-Doublet Model (2HDM), which has received substantial attention in the literature [1]. However, very little is found in the literature on models with more than two Higgs doublets and, in particular, there exists no comprehensive analysis of these models. It is the purpose of this work to study the general Multi-Higgs-Doublet Model (MHDM), and perform a comprehensive analysis of the charged Higgs sector of the model. In the lack of direct experimental knowledge, we study indirect effects of charged scalars in order to place constraints on this sector. In particular, we consider charged scalar contributions to low-energy processes: neutral meson mixing, rare $K$ and $B$ decays, $Z$ boson interactions and $\mathrm{CP}$ violating effects. The results from this combined analysis are used to find the region in parameter space which is consistent with all data. We then discuss the differences between the MHDM and the 2HDM. Throughout this work we assume that there are no significant cancellations with other types of new physics.

Our work is organized as follows. In chapter 2 we describe the MHDM and prove general relations between the model parameters. In chapter 3 we discuss the relevant processes, and list the formulae and numerical data used in our analysis. In chapter 4 we combine all the constraints and find the allowed range of the model parameters. In chapter 5 we discuss the differences between the MHDM and the 2HDM. Finally, chapter 6 contains a summary of our work.

\section{The Model}

\subsection{Introduction}

There are basically two major constraints on any extension of the Higgs sector of the SM. First, it is an experimental fact that $\rho=m_{W}^{2} /\left(m_{Z}^{2} \cos ^{2} \theta_{W}\right)$ is very close to 1 [2]. It is known [1] that in a model with only Higgs doublets, the tree-level value of $\rho=1$ is automatic without adjustment of any parameters in the model. Second, there are strong experimental limits on flavor-changing neutral currents (FCNC's). In the SM, tree-level FCNC's are absent because fermion mass matrices and Higgs-fermion couplings are simultaneously diagonalized. In general, this ceases to be true in a model with a non-minimal Higgs sector. An elegant way to avoid this problem is based on a theorem by Glashow and Weinberg [3] called Natural Flavor Conservation (NFC): tree-level FCNC's mediated by Higgs bosons will be absent if all fermions of a given electric charge couple to no more than one Higgs doublet. This can be achieved by imposing extra symmetries. If we adopt this mechanism, the Higgs couplings to fermions are constrained but not unique. There are five possibilities to couple the Higgs doublets to the known three types of massive fermions (up-type quarks, down-type quarks and charged leptons.). Summary of these possibilities is given in Table 1 1. For Model III at least three Higgs doublets are needed, for Model I one is enough, while for the other models two are sufficient. 


\begin{tabular}{l|ccccc|}
\multicolumn{5}{c}{} & \multicolumn{5}{c}{ Model } \\
\cline { 2 - 6 } d (down-type quarks) & $I$ & $I$ & $I I$ & $I I$ & $I I I$ \\
\cline { 2 - 6 } u (up-type quarks) & 1 & 1 & 1 & 1 & 1 \\
e (charged leptons) & 1 & 1 & 2 & 2 & 2 \\
1 & 2 & 2 & 1 & 3 \\
\cline { 2 - 6 } & & & &
\end{tabular}

Table 1: Summary of all the possibilities of MHDM. The numbers in the table show which Higgs doublet couples to which fermion type.

It does, of course, make a difference if the number of Higgs doublets is larger than the minimal one.

For a general MHDM the Yukawa interactions are given by

$$
-\mathcal{L}_{Y}=\bar{Q}_{L_{i}} F_{i j}^{D} \Phi_{d} D_{R_{j}}+\bar{Q}_{L_{i}} F_{i j}^{U} \tilde{\Phi}_{u} U_{R_{j}}+\bar{L}_{L_{i}} F_{i j}^{E} \Phi_{e} E_{R_{j}}+\text { h.c. }
$$

Where left-handed quark doublets are denoted by $Q_{L_{i}}$, and left-handed lepton doublets by $L_{L_{i}}$. Righthanded quark singlets are denoted by $D_{R_{i}}$ and $U_{R_{i}}$, and right handed charged lepton singlets by $E_{R_{i}}$. The sub-index $i$ is a generation index $(i=1,2,3)$. Higgs doublets are denoted by $\Phi_{j}$ (where $j$ runs from 1 to $n$, the number of Higgs doublets), and $\tilde{\Phi}_{j}=i \sigma_{2} \Phi_{j}^{*}$. Sub-indices $d, u$ and $e$ denote the Higgs doublet that couples to the down-type quarks, up-type quarks and charged leptons, respectively. $F^{U}$ and $F^{D}$ are general $3 \times 3$ Yukawa matrices and we can choose a basis where one of them is real and diagonal. Since we consider massless neutrinos, $F^{E}$ can be chosen real and diagonal. In a general MHDM, with $n$ Higgs doublets, there are $2 n$ charged and $2 n$ neutral scalar fields. After spontaneous symmetry breaking two charged fields and one neutral field become the would-be Goldstone boson "eaten" by the $W^{ \pm}$and the $Z$ in order to acquire their masses, and only $2(n-1)$ charged and $2 n-1$ neutral physical scalars are left. The Yukawa interaction of the physical charged scalars with fermion mass eigenstates is given by 纯

$$
\mathcal{L}_{Y}^{ \pm}=\left(2 \sqrt{2} G_{F}\right)^{1 / 2} \sum_{i=2}^{n}\left(X_{i} \bar{U}_{L} V M_{D} D_{R}+Y_{i} \bar{U}_{R} M_{U} V D_{L}+Z_{i} \bar{N}_{L} M_{E} E_{R}\right) H_{i}^{+}+\text {h.c. }
$$

$M_{D}, M_{U}$ and $M_{E}$ are the diagonal mass matrices of down-type quarks, up-type quarks and charged leptons, respectively. $\mathrm{H}_{i}^{+}$denote the positively charged physical scalars. Left-handed neutrino fields are denoted by $N_{L}$, and the CKM matrix by $V . X_{i}, Y_{i}$ and $Z_{i}$ are complex coupling constants that arise from the mixing matrix for charged scalars. An important feature of the charged scalar fermion interactions is that they are proportional to the fermion masses. Hence, effects are stronger in processes involving heavy fermions, real or virtual. 


\subsection{Relations between $X_{i}, Y_{i}$ and $Z_{i}$}

$X_{i}, Y_{i}$ and $Z_{i}$ arise from the mixing matrix $U$, which rotates the charged scalar interaction eigenstates into the mass eigenstates $[4$

$$
\left(\begin{array}{c}
G^{+} \\
H_{2}^{+} \\
\vdots \\
H_{n}^{+}
\end{array}\right)=U\left(\begin{array}{c}
\Phi_{1}^{+} \\
\Phi_{2}^{+} \\
\vdots \\
\Phi_{n}^{+}
\end{array}\right)
$$

where $G^{+}$is the would-be Goldstone boson and $H_{i}^{+}$are the physical charged scalars. $U$ is an $n \times n$ unitary matrix that can be parameterized as the generalized CKM matrix with $n(n-1) / 2$ angles and $(n-1)(n-2) / 2$ phases [4]. Since $G^{+}$is the Goldstone boson that gives the $W^{+}$its mass in the same way as in the SM, we obtain

$$
G^{+}=\frac{1}{v} \sum_{i=1}^{n} v_{i} \Phi_{i}^{+}
$$

where $v_{i} \equiv<\Phi_{i}>$ and $v \cong 246 \mathrm{GeV}$ is the $\mathrm{SM}$ vacuum expectation value (vev). Using eq. (3) we get

$$
v_{i}=U_{1 i} v, \quad \sum_{i=1}^{n} v_{i}^{2}=v^{2} .
$$

We define

$$
X_{i}=\frac{U_{d i}}{U_{d 1}}, \quad Y_{i}=-\frac{U_{u i}}{U_{u 1}}, \quad Z_{i}=\frac{U_{e i}}{U_{e 1}} .
$$

Using these definitions and the unitarity of $U$, we obtain the following relations:

\section{Relation 1}

$$
\begin{array}{cc}
\sum_{i=2}^{n} X_{i} Y_{i}^{*}=1 & \left(\text { for } \Phi_{d} \neq \Phi_{u}\right), \\
\sum_{i=2}^{n} X_{i} Z_{i}^{*}=-1 & \left(\text { for } \Phi_{d} \neq \Phi_{e}\right), \\
\sum_{i=2}^{n} Y_{i} Z_{i}^{*}=1 & \left(\text { for } \Phi_{u} \neq \Phi_{e}\right) .
\end{array}
$$

\section{Relation 2}

$$
\sum_{i=2}^{n}\left|X_{i}\right|^{2}=\frac{v^{2}}{v_{d}^{2}}-1, \quad \sum_{i=2}^{n}\left|Y_{i}\right|^{2}=\frac{v^{2}}{v_{u}^{2}}-1, \quad \sum_{i=2}^{n}\left|Z_{i}\right|^{2}=\frac{v^{2}}{v_{e}^{2}}-1
$$

Relation $3 X_{i}, Y_{j}$ and $Z_{k}(i, j, k \in 2 . . n)$ cannot be simultaneously large (more than $\sqrt{n-1}$ ), unless there is at least one Higgs doublet that does not couple to fermions. 
These relations are proved in appendix A.

Special cases of our results are known for 2HDM and 3HDM. In 2HDM there is only one charged scalar, so we drop the sub-indices of $X_{i}, Y_{i}$ and $Z_{i}$. For Model II [1]

$$
Z=X=\tan \beta, \quad Y=\cot \beta, \quad \tan \beta=\frac{v_{u}}{v_{d}},
$$

and the three relations are clearly fulfilled. For 3HDM [5]

$$
X_{1} Y_{1}^{*}=1-X_{2} Y_{2}^{*}, \quad Y_{1}^{2}+Y_{2}^{2}=\frac{v_{d}^{2}+v_{e}^{2}}{v_{u}^{2}}, \quad X_{1}^{2}+X_{2}^{2}=\frac{v_{u}^{2}+v_{e}^{2}}{v_{d}^{2}},
$$

which are examples of relations 11 and 2 .

A few points are in order:

1. An important relation between $X$ and $Y$ in $2 \mathrm{HDM}$ (Model $I I$ ), namely $X Y^{*}=1$, is not retained in the framework of a general MHDM. Consequently, processes involving this combination can be enhanced or suppressed in MHDM compared to 2HDM. A similar statement holds for the combinations $X Z^{*}$ and $Y Z^{*}$.

2. The most general features of MHDM can be realized in 4HDM. In 3HDM (Model III) the couplings cannot be simultaneously large. This constraint is removed in 4HDM. However, in most of the processes that we study, not all couplings are involved, and 3HDM is general enough.

3. From relation 1 we see that if all charged scalars were degenerate, the model practically reduces to $2 \mathrm{HDM}$, namely, the relations $X Y^{*}=1, X Z^{*}=-1$ and $Y Z^{*}=1$ hold. Consequently, in order to exploit the most general MHDM, we assume that one of the charged scalars is much lighter than the others, and that all the heavy charged scalars effectively decouple from the fermions. Then, we consider in the calculations the single light charged scalar, and drop the sub-indices of $H_{i}, X_{i}, Y_{i}$ and $Z_{i}$.

\subsection{CP violation}

Within the framework of MHDM, CP violation could arise in charged scalar exchange if there are at least three Higgs doublets [6]. Then, there are two mechanisms which give rise to CP violation (for a review see [5]):

1. Spontaneous CP violation (SCPV). CP symmetry is enforced at the Lagrangian level, but broken through complex vev's. The requirement of SCPV forces $\delta_{\mathrm{CKM}}=0$ [7]. In this case, CP noninvariance arises solely from charged scalar exchange. However, experimental data exclude this possibility [8, 9].

2. Explicit $\mathrm{CP}$ violation. $\mathrm{CP}$ is broken by complex Yukawa couplings and possibly by complex vev's巴. In such a case, $\mathrm{CP}$ violation can arise from both charged scalar exchange and $W^{ \pm}$ exchange.

\footnotetext{
${ }^{1}$ Actually, CP can also be broken through complex scalar couplings. We will not discuss this possibility.
} 
Figure 1: The Feynman rules for the charged scalar - fermions interaction within MHDM.

In both cases, $\mathrm{CP}$ violation in the Higgs sector is manifest in phases that appear in the combinations $X Y^{*}, X Z^{*}$ and $Y Z^{*}$. We emphasize that the requirement that the charged scalars are nondegenerate is mandatory for $\mathrm{CP}$ violation from the Higgs sector. If the charged scalars were degenerate, $X Y^{*}$ is replaced by $\sum_{i=2}^{n} X_{i} Y_{i}^{*}=1$, which is real.

\subsection{Summary}

The model that we study is the SM with extended Higgs sector. Its Higgs sector contains more than two Higgs doublets with NFC. We focus on the charged scalars, and assume that all but the lightest of the charged scalars effectively decouple from fermions. The couplings of the lightest charged scalar to fermions are given by:

$$
\mathcal{L}_{Y}^{+}=\left(2 \sqrt{2} G_{F}\right)^{1 / 2}\left(X \bar{U}_{L} V M_{D} D_{R}+Y \bar{U}_{R} M_{U} V D_{L}+Z \bar{N}_{L} M_{E} E_{R}\right) H^{+}+\text {h.c. }
$$

This interaction yields the Feynman rules given in fig 1. In this model there are several new parameters: The mass of the lightest charged scalar: $m_{H}$, and the coupling constants: $X, Y$ and $Z$. These couplings are arbitrary complex numbers. It is the subject of the coming chapters to find the experimentally allowed ranges for these parameters.

\section{Phenomenology}

In this chapter we discuss various processes where a virtual charged scalar may contribute significantly. From the experimental results for these processes, we are able to constrain the parameters of the model. Wherever possible we use the experimental data at the 95\% CL. For theoretical uncertainties we use estimates and cannot assign a confidence level to the errors. Before discussing the processes, we list values of quark sector parameters that we use, and give constraints on the charged scalar parameters that are obtained from direct measurements and perturbativity.

\subsection{Values of quark parameters}

For quark masses we use [10]

$$
m_{u}=5 \mathrm{MeV}, \quad m_{d}=9 \mathrm{MeV}, \quad m_{s}=180 \mathrm{MeV}, \quad m_{c}=1.4-1.5 \mathrm{GeV}, \quad m_{b}=4.6-5 \mathrm{GeV} .
$$

For the top quark the situation is different. First, it is the only quark that has not been observed. Thus, there is large uncertainty in its mass. Second, since it is the heaviest quark, its coupling to the charged scalar is the largest. The direct search for the top at CDF gives $m_{t}>113 \mathrm{GeV}$ [11], while the electroweak precision measurements at LEP give $m_{t}<185 \mathrm{GeV}$ [12]. Within the framework of MHDM both bounds are relaxed. If $m_{H}<m_{t}$, the top may escape detection at CDF [13]. Additional loops that include the extra scalars may affect the electroweak precision measurements [14. Then, 
the bounds on $m_{t}$ are related to the MHDM parameters. Thus, whenever we use some values of the MHDM parameters we use only values of $m_{t}$ that are consistent with them.

For the values of the CKM matrix elements we use [15, 16, 17]

$$
\begin{aligned}
\left|V_{u d}\right| \cong\left|V_{c s}\right| \cong\left|V_{t b}\right| & \cong 1 \\
\left|V_{c d}\right| \cong\left|V_{u s}\right| & \cong 0.22 \\
\left|V_{t s}\right| \cong\left|V_{c b}\right| & =0.040 \pm 0.007 \\
\left|V_{u b} / V_{c b}\right| & =0.08 \pm 0.03
\end{aligned}
$$

Unitarity constraints on $\left|V_{t d}\right|$ give

$$
\left|V_{t d}\right|=0.004-0.016
$$

The allowed ranges for $\left|V_{t d}\right|$ and $\delta_{\mathrm{CKM}}$ are different in the SM and in MHDM [18]. Furthermore, they are related and depend on $m_{t}$.

For the masses of the physical particles we use the values quoted by the Particles Data Group (PDG) [2].

\subsection{Constraints on scalar parameters}

For the mass of the lightest charged scalar we use [19]

$$
m_{H}>43 \mathrm{GeV} \text {. }
$$

There is no upper bound on $m_{H}$ : The SM Higgs boson is bounded by unitarity to be lighter than about $1 \mathrm{TeV}$, but there is no similar unitarity bound on the charged scalar of MHDM (for a discussion of this point see [1]).

We can get perturbativity bounds on $|X|,|Y|$ and $|Z|$ : We restrict ourselves to the range where perturbation expansion is valid. Following [20] we find

$$
\begin{aligned}
& |Y|<\frac{600 \mathrm{GeV}}{m_{t}}<7, \\
& |X|<\frac{600 \mathrm{GeV}}{m_{b}} \approx 130, \\
& |Z|<\frac{600 \mathrm{GeV}}{m_{\tau}} \approx 340 .
\end{aligned}
$$

There is no lower bound on $|X|,|Y|$ and $|Z|$. When all of them vanish, the model practically reduces to the SM.

\section{3 $\quad B-\bar{B}$ mixing}

The mass difference in the neutral $B$ meson system $\left(\Delta m_{B}\right)$ has been measured and it fits the SM predictions [21, 15, 22]. However, there are large uncertainties in the calculation (mainly in the values 
Figure 2: The diagrams that contribute to $B-\bar{B}$ mixing. (a): The SM diagram. (b): The two-Higgs diagram. (c): The mixed diagrams. The diagrams where the intermediate bosons and fermions are exchanged are not shown.

of $m_{t}, V_{t d}$ and $f_{B}$ ) that leave a lot of room for non-SM contributions. The MHDM extra contributions to the mixing come from box diagrams with intermediate charged scalars (see fig. 2).

In the calculation we neglect external momenta but keep all quark masses. We use the Vacuum Insertion Approximation (VIA) which is a good approximation in this case [23]. For intermediate quark only the top quark is important

$$
M_{12}\left(B_{q}\right)=\frac{G_{F}^{2}}{64 \pi^{2}} m_{W}^{2} \eta_{B}\left(V_{t q}^{*} V_{t b}\right)^{2}\left[I_{W W}+I_{H H}+I_{H W}\right] \quad q=d, s .
$$

The SM contribution (fig. 2(a)) is $I_{W W}$ [21], while box diagrams with two charged scalar propagators (fig. 2(b)) or one charged scalar and one $W$ propagator (fig. 2(c)) give $I_{H H}$ and $I_{H W}$, respectively. $\eta_{B} \sim 0.55$ is a QCD correction factor [24]. The QCD corrections to the MHDM diagrams have been calculated in the leading logarithmic approximation [25], and only for the terms with the same Dirac structure as the SM diagrams. However, following [20] we apply the same QCD correction factor both to the SM and the MHDM contributions. In what follows we present only the most important terms:

$$
\begin{aligned}
I_{W W} & =x_{t} 4 I_{0}\left(x_{t}\right) V_{L L}, \\
I_{H H} & =x_{t}\left[y_{t}|Y|^{4} I_{1}\left(y_{t}\right) V_{L L}+y_{t} y_{b}\left(X Y^{*}\right)^{2} 4 I_{2}\left(y_{t}\right) S_{L L}+y_{b} y_{q}|X|^{4} I_{1}\left(y_{t}\right) V_{L L}\right], \\
I_{H W} & =x_{t}\left[x_{t}|Y|^{2}\left(2 I_{3}\left(x_{t}, x_{H}\right)-8 I_{4}\left(x_{t}, x_{H}\right)\right) V_{L L}+2 x_{t} x_{b}\left(Y X^{*}\right) I_{4}\left(x_{t}, x_{H}\right) S_{L L}\right],
\end{aligned}
$$

where $x_{q} \equiv m_{q}^{2} / m_{W}^{2}$ and $y_{q} \equiv m_{q}^{2} / m_{H}^{2}$. The matrix elements, $V_{L L}$ and $S_{L L}$, and the loop integrals, $I_{i}$, are given in appendix B. Setting $m_{b}$ and $m_{d}$ to zero we get the known results [26, 27], while for the case where only $m_{d}$ is set to zero our results agree with those of [8]. We use $\Delta m_{B}=2\left|M_{12}\right|$ and 2, 28, 17]

$$
x_{d} \equiv \Delta m_{B} / \Gamma_{B}=0.67 \pm 0.11, \quad f_{B}=190 \pm 50 \mathrm{MeV}, \quad \tau_{B}=1.49 \pm 0.03 \text { ps. }
$$

We conclude that large contribution to $B-\bar{B}$ mixing may arise for large $Y$ or large $X Y^{*}$. For the $B$ system the contribution from the term proportional to $|X|^{4}$ is small, even for $X$ as large as the perturbativity bound. However, for the $B_{s}$ system its contribution can be large.

\section{$3.4 \quad K-\bar{K}$ mixing}

There are two well measured quantities related to $K-\bar{K}$ mixing: the mass difference $\Delta m_{K}$ and the $\mathrm{CP}$ violating observable $\epsilon$ (for a review see [15]). Taking into account the bounds from $B-\bar{B}$ mixing, we find that charged scalar mediated diagrams may give only a small contribution to $\Delta m_{K}$ [1]. The situation is different for $\epsilon$ [22]

$$
|\epsilon|=\frac{\operatorname{Im}\left(M_{12}\right)}{\sqrt{2} \Delta M}
$$


$\epsilon$ is a very accurately measured quantity, but there are large uncertainties in the SM calculation (mainly from $m_{t}, V_{t d}$ and $B_{K}$ ). In MHDM there are two new sources that can contribute to $\epsilon$ : The imaginary part of the charged scalar box diagrams, and enhanced long-distance contributions. The short-distance diagrams are similar to those of $B-\bar{B}$ mixing (fig. 2) with the replacement $b \rightarrow s$. For the SM box diagrams we consider both charm and top intermediate quarks, while for the charged scalar box diagrams only the top quark is important. We keep only the terms that are proportional to the internal quark masses:

$$
\left|\epsilon_{S D}\right|=\frac{1}{\sqrt{2} \Delta M} \frac{G_{F}^{2}}{48 \pi^{2}} m_{W}^{2} m_{K} f_{K}^{2} B_{K} \operatorname{Im}\left[I^{c}+I^{t}+I^{c t}\right],
$$

with

$$
\begin{aligned}
I^{c} & =\eta_{1} x_{c} \lambda_{c}^{2} I_{0}\left(x_{c}\right), \\
I^{t} & =\eta_{2} x_{t} \lambda_{t}^{2}\left[I_{0}\left(x_{t}\right)+y_{t}|Y|^{4} I_{1}\left(y_{t}\right)+x_{t}|Y|^{2}\left(2 I_{3}\left(x_{t}, x_{H}\right)-8 I_{4}\left(x_{t}, x_{H}\right)\right)\right], \\
I^{c t} & =2 \eta_{3} x_{c} \lambda_{t} \lambda_{c} I_{6}\left(x_{c}, x_{t}\right),
\end{aligned}
$$

where $\lambda_{q}=V_{q s} V_{q d}^{*}$, and $\eta_{1} \sim 0.85, \eta_{2} \sim 0.62$ and $\eta_{3} \sim 0.36$ are QCD corrections factors [25]. Due to the lightness of the valance quarks, the terms that depend on their masses are neglected. This implies that the term that may have introduced $\mathrm{CP}$ violation from the Higgs sector, namely, the term that depends on $X Y^{*}$, is very small and cannot contribute significantly to $\epsilon$. Our result agrees with [29]. For the numerical values we use [2, 22]

$$
|\epsilon|=2.26 \times 10^{-3}, \quad f_{K}=161 \mathrm{MeV}, \quad B_{K}=2 / 3 \pm 1 / 3 .
$$

The long-distance contributions to $\epsilon$ are negligible in the SM but in MHDM, where they depend on the phases of the Higgs sector, they may be important (see [22, 30, 8]). The largest contribution comes from the intermediate $\eta^{\prime}$ [31, 22]

$$
\left|\epsilon_{L D}\right| \approx 0.02 \mathrm{GeV}^{2} \frac{\operatorname{Im}\left(X Y^{*}\right)}{m_{H}^{2}}\left[\ln \frac{m_{H}^{2}}{m_{c}^{2}}-1.5\right] .
$$

There is an additional CP violating parameter, $\epsilon^{\prime} / \epsilon$. There is no unambiguous evidence for $\epsilon^{\prime} / \epsilon \neq$ 0 [2]. Furthermore, there are large uncertainties in its calculation. Therefore, at present, it is not possible to extract useful constraints from it. A discussion can be found in [20, 18].

We conclude that large contribution to $\epsilon$ may arise for large $Y$ through the short-distance contributions, or for large $\operatorname{Im}\left(X Y^{*}\right)$ through the long-distance contributions.

\section{5 $\quad D-\bar{D}$ mixing}

The mixing in the $D$ system is different from other neutral meson systems:

1. The intermediate quarks in the box diagrams are down-type. Consequently, the box diagrams contribution to the mixing is smaller than in mesons where the intermediate quarks are uptype [32]. 
2. $D-\bar{D}$ mixing has not been observed, and only an upper bound has been established [2],

$$
\Delta m_{D}<1.5 \times 10^{-13} \mathrm{GeV} \quad 95 \% \mathrm{CL} .
$$

3. The long distance contribution to the mixing is probably dominant over the SM box diagram. It is estimated to be about two orders of magnitude below the experimental bound [33] or even smaller [34].

Because of the above reasons, $D-\bar{D}$ mixing constrains neither the SM parameters [15], nor (as we find) the MHDM parameters.

The diagrams that contribute are similar to those of $B-\bar{B}$ mixing (fig. 2) with the replacements $b \rightarrow c, d \rightarrow u$ and $t \rightarrow s, b$. In the calculation we neglect the SM contribution and the mixed diagrams, while from the two-Higgs box diagram we take only the term proportional to $|X|^{4}$

$$
M_{12}=\frac{G_{F}^{2}}{48 \pi^{2}} m_{W}^{2} f_{D}^{2} m_{D} I_{H H}
$$

with

$$
I_{H H}=|X|^{4} x_{H}\left[\lambda_{s}^{2} y_{s}^{2} I_{1}\left(y_{s}\right)+\lambda_{b}^{2} y_{b}^{2} I_{1}\left(y_{b}\right)+2 \lambda_{b} \lambda_{s} y_{b} y_{s} I_{5}\left(y_{b}, y_{s}\right)\right]
$$

where $\lambda_{q}=V_{c q} V_{u q}^{*}$. We can simplify the expression using

$$
I_{1}\left(y_{s}\right) \cong I_{1}\left(y_{b}\right) \cong I_{5}\left(y_{b}, y_{s}\right) \cong 1
$$

and get

$$
M_{12}=\frac{G_{F}^{2}}{48 \pi^{2}} m_{W}^{2} f_{D}^{2} m_{D}|X|^{4} x_{H}\left[\lambda_{s}^{2} y_{s}^{2}+\lambda_{b}^{2} y_{b}^{2}+2 \lambda_{b} \lambda_{s} y_{b} y_{s}\right] .
$$

Setting $\lambda_{b}$ to zero, our result agrees with [26], while setting $m_{s}$ to zero, our result agrees with [20]. For the numerical values we use [2, 28]

$$
\Delta m_{D}<1.5 \times 10^{-13} \mathrm{GeV}, \quad f_{D}=170 \pm 30 \mathrm{MeV} .
$$

We conclude that large contribution to $D-\bar{D}$ mixing may arise for large $X$.

\section{6 $Z \rightarrow b \bar{b}$}

The decay $Z \rightarrow b \bar{b}$ has been measured at LEP. Within MHDM, extra contributions arise via virtual scalars in the vacuum polarization diagrams and the vertex corrections. Recently, a new method of analyzing the electroweak precision data was proposed [35]. In this method the non-SM effects manifest themselves via four observables: $\epsilon_{1}, \epsilon_{2}, \epsilon_{3}$ and $\epsilon_{b}$. We are interested in $\epsilon_{b}$ which is closely related to the vertex correction to $Z \rightarrow b \bar{b}$. By comparing the experimentally measured quantity, $\epsilon_{b}$, to the theoretical calculation of the vertex correction, $\nabla_{b}$, one can put bounds on the MHDM parameters [36]

$$
\epsilon_{b} \cong 2.3 \nabla_{b}
$$


Figure 3: The additional MHDM diagrams that contribute to $b \rightarrow s \gamma$. The SM diagrams obtained by replacing the charged scalar propagators with a $W$-boson ones.

$\nabla_{b}$ is defined in [37]:

$$
\nabla_{b}=\nabla_{b}^{S M}+\nabla_{b}^{H}
$$

with

$$
\begin{aligned}
\nabla_{b}^{S M} & \cong-\frac{20 \alpha}{13 \pi}\left(\frac{m_{t}^{2}}{m_{Z}^{2}}\right), \\
\nabla_{b}^{H} & \cong C \cdot F_{L}\left(m_{t}\right)|Y|^{2} m_{t}^{2},
\end{aligned}
$$

where $C$ is given in appendix B and $F_{L}$ can be found in [37]. For the numerical data we use [35]

$$
\epsilon_{b}>-1 \times 10^{-2} \quad 95 \% \text { CL. }
$$

There are also terms in $\nabla_{b}^{H}$ proportional to $\left|m_{b} X\right|^{2}$. Some of these terms depend on the neutral Higgs parameters, but appear with a sign opposite to the terms that depend on the charged Higgs parameters [38]. Thus, cancellation may occur and a bound on $X$ cannot be obtained. This also applies to the decay $Z \rightarrow \tau \bar{\tau}$. There, the MHDM contribution is proportional to $\left|m_{\tau} Z\right|^{2}$, but the terms that depend on the neutral Higgs parameters may cancel the terms that depend on the charged Higgs parameters, and a bound on $Z$ cannot be obtained.

We conclude that large contribution to $Z \rightarrow b \bar{b}$ may arise for large $Y$ or large $X$. However, a bound can be obtained only on $Y$.

\section{$3.7 \quad b \rightarrow s \gamma$}

Recently, the CLEO collaboration has given an upper bound on the radiative $B$ decay 39]

$$
\mathrm{BR}(b \rightarrow s \gamma)<5.4 \times 10^{-4} \quad 95 \% \mathrm{CL},
$$

which is only about a factor of 2 above the SM prediction. Uncertainties in the calculation come mainly from the value of $m_{t}$ 40. The diagrams that contribute to $b \rightarrow s \gamma$ are given in fig 3 . The SM calculation was done in 41] and the MHDM calculation in [42, 43, 8, 44]:

$$
\mathrm{BR}(b \rightarrow s \gamma)=C\left|\eta_{2}+G_{W}\left(x_{t}\right)+\left(|Y|^{2} / 3\right) G_{W}\left(y_{t}\right)+\left(X Y^{*}\right) G_{H}\left(y_{t}\right)\right|^{2},
$$

where

$$
C \equiv \frac{3 \alpha \eta_{1}^{2} \operatorname{BR}\left(B \rightarrow X_{c} \ell \nu\right)}{2 \pi F_{p s}\left(m_{c}^{2} / m_{b}^{2}\right)} \approx 3 \times 10^{-4} .
$$

$F_{p s} \sim 0.5$ is a phase space factor given in appendix B, $\eta_{1} \sim 0.66$ and $\eta_{2} \sim 0.57$ are QCD correction factors 44]. The expressions for the $G$-functions are given in appendix B. The SM result can be obtained by setting $X$ and $Y$ to zero in (36). In the full calculation [15] a term proportional to $|X|^{2}$ appears, but it is suppressed by $\left(m_{s} / m_{b}\right)^{2}$ and we can safely drop it.

We conclude that large contribution may arise for large $Y$ or large $X Y^{*}$. 
Figure 4: The diagrams that contribute to $K^{+} \rightarrow \pi^{+} \nu \bar{\nu}$. (a) The $Z$ penguin diagrams. The solid square represent the effective $s d Z$ vertex which induced by diagrams similar to these of $b \rightarrow s \gamma$ (fig. (3). (b) The SM box diagram. (c) The two charged scalars box diagram. (d) The mixed diagram. The second mixed diagram is not shown.

\section{$3.8 K^{+} \rightarrow \pi^{+} \nu \bar{\nu}$}

The branching ratio of the decay $K^{+} \rightarrow \pi^{+} \nu \bar{\nu}$ has not yet been measured. The current bound is 46]

$$
\mathrm{BR}\left(K^{+} \rightarrow \pi^{+} \nu \bar{\nu}\right)<6.8 \times 10^{-9} \quad 95 \% \mathrm{CL},
$$

is about two orders of magnitude above the SM prediction. The calculation suffers from uncertainties in $m_{t}$ and $V_{t d}$ (for a review see [47]). The diagrams that contribute to this process are given in fig. 4 . In the SM, the penguin and the box diagrams are of the same order, and intermediate charm and top quarks are important. In diagrams that involve charged scalars the intermediate top quark is dominant. Since the external quark masses are small, we consider only terms proportional to internal quark masses.

The SM diagrams were calculated in 41. The MHDM result is obtained from the 2HDM calculation [20, 18, 48] by multiplying the two charged scalars box diagram by $|Y Z|^{2}$ and the mixed diagrams by $\operatorname{Re}\left(Y Z^{*}\right)$

$$
\mathrm{BR}\left(K^{+} \rightarrow \pi^{+} \nu \bar{\nu}\right)=\frac{C}{\left|V_{u s}\right|^{2}} \sum_{\ell=e, \mu, \tau}\left|I^{S M}+I^{H H}+I^{W H}+I^{Z H}\right|^{2},
$$

with

$$
C \equiv \frac{G_{F}^{2} m_{W}^{4}}{4 \pi^{4}} \operatorname{BR}\left(K^{+} \rightarrow \pi^{0} e^{+} \nu\right) \approx 6.9 \times 10^{-7} .
$$

The SM contribution is $I^{S M}$, the two charged scalars box diagram is $I^{H H}$, the mixed diagrams are $I^{W H}$ and the charged scalar penguin diagrams are $I^{Z H}$

$$
\begin{aligned}
I^{S M} & =\eta_{c} \lambda_{c} D_{S M}\left(x_{c}, x_{\ell}\right)+\lambda_{t} D_{S M}\left(x_{t}, x_{\ell}\right), \\
I^{H H} & =|Y Z|^{2} \lambda_{t} x_{t} y_{\ell} I_{5}\left(y_{t}, y_{\ell}\right) \\
I^{W H} & =\operatorname{Re}\left(Y Z^{*}\right) \lambda_{t} x_{t} x_{\ell}\left(I_{7}\left(x_{t}, x_{\ell}, x_{H}\right)+I_{8}\left(x_{t}, x_{\ell}, x_{H}\right)\right), \\
I^{Z H} & =|Y|^{2} \lambda_{t} D_{Z H}\left(x_{t}, y_{t}\right),
\end{aligned}
$$

where $\lambda_{q}=V_{q d} V_{q s}^{*}$ and the $D$-functions are given in appendix B. $\eta_{c} \sim 0.7$ is a QCD correction factor [47, while QCD corrections to the diagrams with an intermediate top quark are small 49. While for the penguin diagrams the MHDM contribution is independent of the final lepton, the charged scalar box diagrams depend on the internal lepton mass and it may be important only for internal $\tau$. In $2 \mathrm{HDM}$ (Model II) $Y Z^{*}=1$ and the charged scalar box diagrams are negligible. Our results disagree with those of [47] where it is claimed that the charged scalar box diagrams are not important even within MHDM. We believe that the reason for this is that they assume that all the 
Figure 5: The diagrams that contribute to $B \rightarrow X \tau \nu_{\tau}$. (a) The SM diagram. (b) The charged scalar mediated diagram.

coefficients in the Higgs potential are of the same order. Then, $|Y Z|=O(1)$ and the effect is indeed small.

A similar calculation applies to the decay $B \rightarrow X_{s} \nu \bar{\nu}$. However, due to the lack of experimental data, no constraints on the parameters can be obtained.

We conclude that large contribution to $K^{+} \rightarrow \pi^{+} \nu \bar{\nu}$ may arise for large $Y$ or large $Y Z^{*}$.

\section{$3.9 \quad B \rightarrow X \tau \nu_{\tau}$}

The branching ratio for $B \rightarrow X \tau \nu_{\tau}$ has been recently measured by the ALEPH collaboration [50] which leads to the upper bound

$$
\mathrm{BR}\left(B \rightarrow X \tau \nu_{\tau}\right)<4.00 \% \quad 95 \% \mathrm{CL}
$$

The uncertainties in the calculation are mainly from $m_{b}$ and $m_{c}$. The diagrams that contribute to the decay are given in fig. 5. In the calculation we neglect charmless final states, since they are highly suppressed by CKM elements. A recent SM calculation was done in [51]. The MHDM result is obtained from the 2HDM calculation [52, 53, 54] by replacing $|X|^{2}$ with $X Z^{*}$

$$
B R\left(B \rightarrow X \tau \nu_{\tau}\right)=B R^{S M}\left(B \rightarrow X \tau \nu_{\tau}\right)\left(1+\frac{1}{4}|R|^{2}-D \cdot \operatorname{Re}(R)\right),
$$

where the SM result is 51

$$
B R^{S M}\left(B \rightarrow X \tau \nu_{\tau}\right)=(2.30 \pm 0.25) \%
$$

and

$$
R \equiv \frac{m_{\tau} m_{b} X Z^{*}}{m_{H}^{2}}, \quad D \equiv 2 \frac{m_{\tau}}{m_{b}} \frac{\tilde{F}_{p s}^{i n t}\left(m_{c} / m_{b}, m_{\tau} / m_{b}\right)}{\tilde{F}_{p s}\left(m_{c} / m_{b}, m_{\tau} / m_{b}\right)}
$$

The phase space functions $\tilde{F}_{p s}$ and $\tilde{F}_{p s}^{i n t}$ are given in appendix B. For the ranges of masses that we use $D \approx 0.43 \pm 0.01$. We disagree with [54] about the numerical value of $D$. Due to this disagreement the bound that we obtain is weaker than the bound obtained by [54]. In 2HDM (Model II) the interference term always reduces the rate, while in MHDM it may also enhance it, depending on the relative phase between $X$ and $Z$. Nevertheless, in order to get bounds on $|X Z|$ one should take the minimal value for the branching ratio, which corresponds to $\arg \left(X Z^{*}\right)=0$. Thus, the bound on $|X|^{2}$ in $2 \mathrm{HDM}$ is the same as the bound on $|X Z|$ in MHDM.

We conclude that large contribution to $B \rightarrow X \tau \nu_{\tau}$ may arise for large $X Z$.

\section{$3.10 \quad e-\mu$ universality in $\tau$ decays}

$e-\mu$ universality is confirmed in leptonic decays of pions and kaons, and in $\tau$ decays (for a review see [55]). The SM and MHDM diagrams are similar to those of $B \rightarrow X \tau \nu_{\tau}$ (fig. 5) with the replacements: $b \rightarrow \tau, c \rightarrow \nu_{\tau}$ and $\tau \rightarrow \ell(\ell=e, \mu)$. At first glance, it seems that a useful bound on the 
MHDM parameters cannot be obtained since the final leptons are very light. However, in purely leptonic decays both the theoretical calculation and the experimental measurement are clean. We then require that the charged scalar contribution does not exceed the difference between the SM prediction and the experimental result. The calculation in the case of muon decay was done in [56] and for $\tau$ decay in 52,57$]^{2}$

$$
\frac{\operatorname{BR}\left(\tau \rightarrow \mu \nu_{\tau} \bar{\nu}_{\mu}\right)}{\operatorname{BR}\left(\tau \rightarrow e \nu_{\tau} \bar{\nu}_{e}\right)}=F_{p s}\left(m_{\mu}^{2} / m_{\tau}^{2}\right)\left[1+\frac{R^{2}}{4}-D \cdot R\right] \cong 0.9726\left[1+\frac{R^{2}}{4}-0.125 R\right],
$$

where

$$
R \equiv \frac{m_{\tau} m_{\mu}|Z|^{2}}{m_{H}^{2}}, \quad D \equiv 2 \frac{m_{\mu}}{m_{\tau}} \frac{F_{p s}^{i n t}\left(m_{\mu}^{2} / m_{\tau}^{2}\right)}{F_{p s}\left(m_{\mu}^{2} / m_{\tau}^{2}\right)} .
$$

$F_{p s}^{\text {int }}$, the phase space function for the interference term, is given in appendix B. We use the recent numerical values [58], which leads to the upper bound

$$
\frac{\operatorname{BR}\left(\tau \rightarrow \mu \nu_{\tau} \bar{\nu}_{\mu}\right)}{\operatorname{BR}\left(\tau \rightarrow e \nu_{\tau} \bar{\nu}_{e}\right) F_{p s}\left(m_{\mu}^{2} / m_{\tau}^{2}\right)}<1.035 \quad 95 \% \mathrm{CL}
$$

We conclude that strong deviation from $e-\mu$ universality in $\tau$ decays may arise for large $Z$.

\subsection{Electric dipole moment of the neutron}

An electric dipole moment (EDM) of an elementary particle is a manifestation of $\mathrm{CP}$ violation (for a review see [22]). In our discussion we concentrate on the EDM of the neutron (NEDM), $D_{n}$. Although there are very large uncertainties in the calculation, we know that the contribution to the NEDM from the electro-weak sector of the SM is much smaller than the current experimental bound [2]

$$
\left|D_{n}\right| \leq 1.2 \times 10^{-25} \text { e cm } 95 \% \text { CL }
$$

A large NEDM can be generated within MHDM in many ways (for a review see [30]). In our discussion we concentrate on the two that involve charged scalars: the EDM of the down quark, and the three gluon operator. The contribution to the NEDM from the EDM of the down quark is given in [59. We neglect the contribution from the internal top quark and using $m_{c} \ll m_{H}$ we get

$$
D_{n}=\frac{\sqrt{2} G_{F}}{9 \pi^{2}} m_{d} \operatorname{Im}\left(X Y^{*}\right)\left|V_{c d}\right|^{2}\left(\ln \left(y_{c}\right)+0.75\right) y_{c}
$$

For the three gluon operator we use the naive dimensional analysis result [60]

$$
D_{n}=\frac{4 \sqrt{2} \zeta G_{F} g_{s}^{3}}{(4 \pi)^{4}} \operatorname{Im}\left(X Y^{*}\right) h^{\prime}\left(y_{t}\right)
$$

where

$$
h^{\prime}(y)=\frac{y}{8(1-y)^{3}}\left[4 y-y^{2}-3-2 \ln (y)\right] .
$$

\footnotetext{
${ }^{2}$ We disagree with the numerical factor of the interference term in eq. (8) of [57]. It seems to us that they divide by $F_{p s}^{i n t} / F_{p s}$ instead of multiplying by this factor.
} 
$\zeta$, the QCD correction factor, depends sensitively on the scale 61]

$$
\zeta=10^{-3}-10^{-1}
$$

We conclude that large contribution to the NEDM may arise for large $\operatorname{Im}\left(X Y^{*}\right)$.

\section{$3.12 \quad B \rightarrow \ell^{+} \ell^{-}$}

The branching ratio for $B \rightarrow \ell^{+} \ell^{-}$has not been measured, but there is an upper bound for $\ell=e, \mu$. The diagrams that contribute to $B \rightarrow \ell^{+} \ell^{-}$are similar to the diagrams that contribute to $K^{+} \rightarrow \pi^{+} \nu \bar{\nu}$ (fig. 目). The photon penguin diagrams vanish because of electromagnetic current conservation. The charged scalar penguin diagrams contribute universally for all leptons. However, the charged scalar box diagrams depend on the lepton mass. Thus, it is important only for $B \rightarrow \tau^{+} \tau^{-}$. We emphasize that in $2 \mathrm{HDM}$ the box diagram is negligible since it is proportional to $|Y Z|^{2}$, which is 1 in Model II, or $O(1)$ in Model I. A recent SM calculation was done in [62] and the 2HDM in 63]. We calculated the box diagram. We neglect terms proportional to external quarks masses, and the QCD correction which is small [62]. We get

$$
\mathrm{BR}\left(B \rightarrow \ell^{+} \ell^{-}\right)=\mathrm{BR}_{S M}\left(B \rightarrow \ell^{+} \ell^{-}\right)\left[1+\frac{G_{Z}^{H}\left(x_{t}, y_{t}, y_{\ell}\right)-G_{b o x}^{H}\left(x_{t}, y_{t}, y_{\ell}\right)}{G^{S M}\left(x_{t}\right)}\right]^{2},
$$

where

$$
\operatorname{BR}_{S M}\left(B \rightarrow \ell^{+} \ell^{-}\right)=\tau_{B} \frac{G_{F}^{4} m_{W}^{4}}{32 \pi^{5}} f_{B}^{2} m_{B} m_{\ell}^{2} \sqrt{1-\frac{4 m_{\ell}^{2}}{m_{B}^{2}}}\left|G^{S M}\left(x_{t}\right) \lambda_{t}\right|^{2},
$$

$\lambda_{q}=V_{q d} V_{q b}^{*}$ and $G_{Z}^{H}$ and $G_{b o x}^{H}$ are the charged scalar penguin and box diagram contributions, respectively:

$$
\begin{aligned}
G_{Z}^{H} & =y_{t} x_{t}|Y|^{2} J\left(y_{t}\right) \\
G_{b o x}^{H} & =\frac{1}{8} x_{t} y_{\ell}\left(|Y Z|^{2} I_{9}\left(y_{t}\right)+2 \operatorname{Re}\left(Y Z^{*}\right) I_{10}\left(x_{t}, x_{H}\right)\right) .
\end{aligned}
$$

$G_{S M}, J$ and $I_{i}$ are given in appendix B. Our results agree with [64], but disagree with [63] about the Dirac structure of the matrix element, and the sign of the first term of $I_{9}$. However, since they work within the framework of $2 \mathrm{HDM}$, where the box diagram is negligible, this disagreement does not have numerical significance.

A few points are in order:

1. The same calculation applies to $B_{s}$ decays with $(d \rightarrow s)$. Consequently, the branching ratio for $B_{s}$ decay is larger by approximately $\left|\frac{V_{t s}}{V_{t d}}\right|^{2}$.

2. It was noted 64, 65] that large effects may arise due to neutral scalar penguin diagrams, even in 2HDM. This contribution depends on neutral Higgs sector parameters and could also be small. We note that the contributions from the charged scalar and from the neutral scalars are independent. 
3. The process $K_{L} \rightarrow \mu^{+} \mu^{-}$gets contributions from diagrams similar to $B \rightarrow \ell^{+} \ell^{-}$. Since the muon is light, the box diagram is negligible. In addition, the long distance contribution dominates. Thus, it is difficult to estimate the charged scalar penguin diagrams contribution. We do not discuss this process. For a detailed discussion in the framework of 2HDM, see [20].

4. The processes $B \rightarrow X_{s} \ell^{+} \ell^{-}$gets contributions from diagrams similar to $B \rightarrow \ell^{+} \ell^{-}$, with the addition of photon penguin diagrams. In $2 \mathrm{HDM}$ the charged scalar box diagrams are negligible [42, 66]. However, in MHDM they may be important for final $\tau$ leptons. Then an interesting situation arises: The $\mathrm{Z}$ penguin depends on $Y$, the photon penguin depends on $Y$ and $Y X^{*}$, and the charged scalar box diagrams depend on $|Y Z|$. The process $B \rightarrow X_{s} \tau^{+} \tau^{-}$then depends on all the charged Higgs parameters. Furthermore, the neutral scalar penguin [64, 65] may also contribute. However, due to the poor experimental data, no bounds can be obtained from these decays.

We conclude that large contribution to $B \rightarrow \ell^{+} \ell^{-}$may arise for large $Y$, and for $B \rightarrow \tau^{+} \tau^{-}$also for large $Y Z$.

\section{$3.13 \quad B \rightarrow \ell \nu$}

The branching ratios of the decays $B \rightarrow \ell \nu(\ell=\mu, \tau)$ have not yet been measured, and the current 90\% CL bounds 67

$$
\begin{aligned}
& \mathrm{BR}(B \rightarrow \mu \nu)<2.0 \times 10^{-5} \\
& \mathrm{BR}(B \rightarrow \tau \nu)<1.2 \%
\end{aligned}
$$

are about two orders of magnitude above the SM predictions. The calculation suffers from uncertainties in $f_{B}$ and $V_{u b}$. The diagrams that contribute to the decays are similar to those of $B \rightarrow X \tau \nu_{\tau}$ (fig. 5), with the replacement $c \rightarrow u$. The MHDM result is obtained from the 2HDM calculation [68] by replacing $|X|^{2}$ by $X Z^{*}$ :

$$
B R(B \rightarrow \ell \nu)=B R^{S M}(B \rightarrow \ell \nu)|1-R|^{2},
$$

where the SM result is

$$
B R^{S M}(B \rightarrow \ell \nu)=\frac{G_{F}^{2} m_{B} m_{\ell}^{2}}{8 \pi}\left(1-\frac{m_{\ell}^{2}}{m_{B}^{2}}\right) f_{B}^{2}\left|V_{u b}\right|^{2} \tau_{B},
$$

and

$$
R \equiv \frac{m_{B}^{2} X Z^{*}}{m_{H}^{2}}
$$

In 2HDM (Model II) the interference term always reduces the rate, while in MHDM it may also enhance it, depending on the relative phase between $X$ and $Z$. Nevertheless, in order to get bounds on $|X Z|$ one should take the minimal value for the branching ratio, which corresponds to $\arg \left(X Z^{*}\right)=0$. Thus, the bound on $|X|^{2}$ in $2 \mathrm{HDM}$ is the same as the bound on $|X Z|$ in MHDM.

We conclude that large contribution to $B \rightarrow \ell \nu$ may arise for large $X Z$. 
Figure 6: The upper bound on $|Y|$ as a function of the lightest charged scalar mass $m_{H}$. The three curves correspond to $m_{t}=100$ (solid), 140 (dashed) and 180 (dotted) GeV.

\section{Combined analysis}

In this chapter we combine the results from the previous chapter and determine the region in parameter space allowed by the data.

Several processes are sensitive to $|Y|$. For some of them there are very poor experimental data: $B_{s}-\bar{B}_{s}$ mixing and $B \rightarrow X_{s} \nu \bar{\nu}$. For others, the experimental bounds are far above the SM predictions: $K^{+} \rightarrow \pi^{+} \nu \bar{\nu}, B \rightarrow \ell^{+} \ell^{-}$and $B \rightarrow X_{s} \ell^{+} \ell^{-}$. The bounds obtained from $\Delta m_{K}$ and $K_{L} \rightarrow \mu^{+} \mu^{-}$are very weak. The decay $b \rightarrow s \gamma$ gives a strong constraint on $|Y|$ in the case of 2HDM (Model II) [69]. In MHDM, however, there is a possibility of cancellation between the two terms that depend on the MHDM parameters (see eq. (36)), and such a bound cannot be obtained. Then, there are three observables that constrain $|Y|: \epsilon$ (eq. (21)), $x_{d}$ (eq. (17)) and $\epsilon_{b}$ (eq. (32)). At present, $\epsilon_{b}$ gives the strongest bound [36]. It is important to note that the bound from $\epsilon_{b}$ is strong since the experimental value is far from the SM prediction. Actually, at the $1 \sigma$ level $m_{t}$ is found to be less than the CDF lower bound. Had the data fitted large $m_{t}$, the bound on $|Y|$ from $B-\bar{B}$ mixing may become the strongest. Our results are given in fig. 6. For example, we obtain

$$
|Y|<1.3 \quad \text { for } m_{t}=140 \mathrm{GeV} \text { and } m_{H}=45 \mathrm{GeV} \text {. }
$$

The only potential constraint on $|X|$ comes from $D-\bar{D}$ mixing (eq. (29)). However, the current experimental bound with the large uncertainties in the CKM elements gives no useful bound. We conclude that $|X|$ is constrained by the perturbativity bound (eq. (16)):

$$
|X|<130 \text {. }
$$

$|Z|$ is constrained by $e-\mu$ universality in $\tau$ decays (eq. (46)) and by the perturbativity bound (eq. (16)). For $m_{H}>175 \mathrm{GeV}$ the perturbativity bound is stronger:

$$
|Z|<\min \left(1.93 m_{H} \mathrm{GeV}^{-1}, 340\right)
$$

$|X Z|$ is constrained by $B \rightarrow X \tau \nu_{\tau}$ (eq. (43)), $B \rightarrow \mu \nu, B \rightarrow \tau \nu$ (eq. (58)) and by the product of the bounds on $|X|$ and $|Z|$. For light charged scalar $B \rightarrow X \tau \nu_{\tau}$ give the strongest bound, while for $m_{H}>370 \mathrm{GeV}$ the perturbativity bound is the strongest:

$$
|X Z|<\min \left(0.32 m_{H}^{2} \mathrm{GeV}^{-2}, 44200\right) .
$$

In 2HDM (Model II), where $X=Z$, eq. (64) gives $|X|<0.56 m_{H} \mathrm{GeV}^{-1}$, which is weaker than the published bound [50, 54]. The difference arises due to the different values calculated for the interference term and the different values taken for the SM branching ratio. 
Figure 7: The upper bound on $|X Y|$ as a function of the lightest charged scalar mass $m_{H}$. The three curves correspond to $m_{t}=100$ (solid), 140 (dashed) and 180 (dotted) GeV.

Figure 8: The upper bound on $\operatorname{Im}\left(X Y^{*}\right)$ as a function of the lightest charged scalar mass $m_{H}$. The three curves correspond to $m_{t}=100$ (solid), 140 (dashed) and 180 (dotted) GeV.

It is important to note that below certain values of $|X Z|$ and $m_{H}$ the destructive interference leads to suppression and a bound cannot be obtained [68]. This happens at $|X Z|<0.18 m_{H}^{2} \mathrm{GeV}^{-2}$ for $B \rightarrow X \tau \nu_{\tau}$ and at $|X Z|<0.07 m_{H}^{2} \mathrm{GeV}^{-2}$ for $B \rightarrow \ell \nu$. We see that although the current bound from $B \rightarrow X \tau \nu_{\tau}$ is stronger, $B \rightarrow \ell \nu$ can potentially give a stronger bound.

The bound on $\operatorname{Re}\left(X Z^{*}\right)$ is the same as the bound on $|X Z|$. However, the bound on the CP violating parameter $\operatorname{Im}\left(X Z^{*}\right)$ is stronger. $\operatorname{Im}\left(X Z^{*}\right)$ can be bounded from the same processes that bound $|X Z|$. For light charged scalar $B \rightarrow X \tau \nu_{\tau}$ give the strongest bound, while for $m_{H}>440 \mathrm{GeV}$ the perturbativity bound is the strongest:

$$
\operatorname{Im}\left(X Z^{*}\right)<\min \left(0.23 m_{H}^{2} \mathrm{GeV}^{-2}, 44200\right)
$$

Several processes are sensitive to $|Y Z|$. For some of them there are very poor experimental data: $B \rightarrow X_{s} \nu \bar{\nu}, B \rightarrow \tau^{+} \tau^{-}$and $B \rightarrow X_{s} \tau^{+} \tau^{-}$. The only measurement that could potentially bound $|Y Z|$ is $K^{+} \rightarrow \pi^{+} \nu \bar{\nu}$ (eq. (39)). However, due to the current experimental bound and the large uncertainties in the CKM elements this bound is weaker than the product of the bounds on $|Y|$ and $|Z|$. The bounds on $\operatorname{Im}\left(Y Z^{*}\right)$ and on $\operatorname{Re}\left(X Y^{*}\right)$ are the same as the bound on $|Y Z|$. For example, we obtain

$$
|Y Z|<110 \quad \text { for } m_{t}=140 \mathrm{GeV} \text { and } m_{H}=45 \mathrm{GeV} \text {. }
$$

The processes that are sensitive to $X Y^{*}$ are $B-\bar{B}$ mixing, $B \rightarrow X_{s} \ell^{+} \ell^{-}$and $b \rightarrow s \gamma$. The strongest bound comes from $b \rightarrow s \gamma$ (eq. (36)). The bound on $|X Y|$ is very sensitive to $\arg \left(X Y^{*}\right)$ and $|Y|$. The weakest upper bound on $|X Y|$ is obtained for maximal $|Y|$ and $\arg \left(X Y^{*}\right)=\pi$. Then, the cancellation between the two terms, the one proportional to $X Y^{*}$ and the one proportional to $|Y|^{2}$, is maximal. Our results are given in fig. 6. For example, we obtain

$$
|X Y|<4 \quad \text { for } m_{t}=140 \mathrm{GeV} \text { and } m_{H}=45 \mathrm{GeV} \text {. }
$$

The bound on $\operatorname{Re}\left(X Y^{*}\right)$ is the same as that on $|X Y|$. However, the bound on the $\mathrm{CP}$ violating parameter $\operatorname{Im}\left(X Y^{*}\right)$ is stronger. $\operatorname{Im}\left(X Y^{*}\right)$ is bounded by CP violating processes: NEDM (eq. (50)) and $\epsilon$ (eq. 24) ), and by CP conserving ones: $B-\bar{B}$ mixing, $B \rightarrow X_{s} \ell^{+} \ell^{-}$and $b \rightarrow s \gamma$. The strongest bound comes from $b \rightarrow s \gamma$ [9]. Our results are given in fig. 8. For example, we obtain

$$
\operatorname{Im}\left(X Y^{*}\right)<2 \quad \text { for } m_{t}=140 \mathrm{GeV} \text { and } m_{H}=45 \mathrm{GeV} \text {. }
$$




\section{Discussion}

In this chapter we discuss the differences between the general MHDM and the widely discussed 2HDM (Model $I I)^{3}$. In 2HDM there are only two parameters in the charged Higgs sector: the mass of the charged scalar, $m_{H}$, and the ratio of the vacuum expectation values, $\tan \beta$. The lower bound on $\tan \beta$ is the same as the bound on $1 /|Y|$ obtained from the decay $Z \rightarrow b \bar{b}$. Since in 2 HDM $X=Z$, the upper bound on $\tan \beta$ is the same as the bound on $\sqrt{|X Z|}$. For intermediate values of $\tan \beta$ the bound on $m_{H}$ is obtained from the decay $b \rightarrow s \gamma$ (see e.g. [40]). There are two main reasons for the differences between MHDM and 2HDM:

- In MHDM there are four parameters in the charged Higgs sector. In $2 \mathrm{HDM}, X Y^{*}=1$ and $X=Z$ so there are only two. Thus, processes that involve combinations of these parameters can be enhanced or suppressed in MHDM compared to 2HDM.

- The bounds on the parameters in MHDM may be weaker than the corresponding bounds in 2HDM. Thus, the possible effects are larger.

\subsection{Bound on $m_{H}$}

In $2 \mathrm{HDM} b \rightarrow s \gamma$ gives a lower bound on $m_{H}$ almost independent of $\tan \beta: m_{H} \gtrsim 100 \mathrm{GeV}$. In MHDM, however, this bound does not hold. This bound has implications for the experimental search of the charged scalar. In LEP200, a charged scalar of mass up to approximately $80 \mathrm{GeV}$ can be found [19]. Consequently, the charged scalar of 2HDM cannot be detected at LEP200 while the charged scalar of MHDM can. Furthermore, the charged scalar of 2HDM decays dominantly to $\tau \nu$ and $c s$. Within MHDM, when $|X|$ is large and $|Y|$ and $|Z|$ are small, the $c b$ channel becomes important. Large background from $W$ decays is expected if $m_{H} \sim m_{W}$. Since the $W$-boson hardly decays into $c b$, the $H^{+} \rightarrow c \bar{b}$ decay mode is easier to detect.

\subsection{Processes that depend on $|X|$ or $|Z|$}

In 2HDM the bound on $X=Z$ is the same as the bound on $\sqrt{|X Z|}$ in MHDM. Consequently, the bounds on $|X|$ and $|Z|$ are weaker within MHDM. Thus, processes that depend on $|X|$ or $|Z|$ may be enhanced within MHDM compared to the 2HDM case:

- The charged scalar box diagrams can saturate the experimental bound on $D-\bar{D}$ mixing. Furthermore, measurable $\mathrm{CP}$ violating effects in the interference of mixing and decay may arise. Intuitively, the reason is that the charged scalar box diagrams introduce dependence on third generation parameters. Based on the discussion in [32] we find that the measured asymmetry

$$
A_{C P} \equiv \frac{N\left[\left(\ell^{-} X\right)_{D}\left(P^{+} P^{-}\right)_{D}\right]-N\left[\left(\ell^{+} X\right)_{D}\left(P^{+} P^{-}\right)_{D}\right]}{N\left[\left(\ell^{-} X\right)_{D}\left(P^{+} P^{-}\right)_{D}\right]+N\left[\left(\ell^{+} X\right)_{D}\left(P^{+} P^{-}\right)_{D}\right]}
$$

\footnotetext{
${ }^{3}$ In this chapter we refer only to Model II of $2 \mathrm{HDM}$.
} 
$\left(P^{+} P^{-}\right.$is a CP eigenstate, e.g. $\pi^{+} \pi^{-}$or $\left.K^{+} K^{-}\right)$can reach its current upper bound

$$
A_{C P} \leq 0.16
$$

Note, that no new source of CP violation beyond the phase of the CKM matrix is required. A recent estimate [70] shows that within one year operation of a Tau-Charm Factory asymmetries of order $1 \%$ are measurable.

- $B_{s}-\bar{B}_{s}$ mixing can be enhanced. The ratio between the two mixing observables, $x_{d}$ and $x_{s}$, is given by:

$$
\frac{x_{s}}{x_{d}}=r \cdot \frac{\left|V_{t s}\right|^{2}}{\left|V_{t d}\right|^{2}} .
$$

Deviations from $r=1$ in eq. (71) are due to flavor $S U(3)$ breaking effects. From eq. (18) we see that when $|X|$ is close to its upper bound and $m_{H}$ to its lower bound, the last term in $I_{H H}$ dominates $x_{s}$ but it is still small for $x_{d}$. Then, $r$ is enhanced. We find that even $r \sim 7$ is possible.

- Violation of $e-\mu$ universality in $\tau$ decays is proportional to $|Z|^{2}$. Since the bound on $|Z|$ is weaker in MHDM compared with $2 \mathrm{HDM}$, the violation can be stronger.

\subsection{Processes that depend on $Y Z^{*}$ or $X Y^{*}$}

While in $2 \mathrm{HDM} X Y^{*}=Y Z^{*}=1$, in MHDM $X Y^{*}$ and $Y Z^{*}$ are, in principle, arbitrary complex numbers. Thus, processes that involve these combinations can be enhanced or suppressed in MHDM compared with 2HDM:

- FCNC decays with third generation leptons in the final state. In such processes the charged scalar box diagrams may be important. They depends on $Y Z^{*}$ and on the lepton mass. Thus, in processes where leptons from the first and second generation are involved the box diagram is negligible. For $K^{+} \rightarrow \pi^{+} \nu \bar{\nu}$ we find that the SM upper bound

$$
\mathrm{BR}^{S M}\left(K^{+} \rightarrow \pi^{+} \nu \bar{\nu}\right)<4 \times 10^{-10},
$$

is modified into

$$
\mathrm{BR}^{M H D M}\left(K^{+} \rightarrow \pi^{+} \nu \bar{\nu}\right)<1.4 \times 10^{-9},
$$

which is more than 3 times weaker than the SM bound. For $B \rightarrow X_{s} \nu \bar{\nu}$ we find that the SM upper bound

$$
\mathrm{BR}^{S M}\left(B \rightarrow X_{s} \nu \bar{\nu}\right)<5.5 \times 10^{-5}
$$

is modified into

$$
\mathrm{BR}^{M H D M}\left(B \rightarrow X_{s} \nu \bar{\nu}\right)<8.5 \times 10^{-4},
$$

which is more than a order of magnitude weaker than the SM bound. A similar enhancement may occur for the decays $B \rightarrow \tau^{+} \tau^{-}, B_{s} \rightarrow \tau^{+} \tau^{-}$and $B \rightarrow X_{s} \tau^{+} \tau^{-}$. 


\begin{tabular}{||c||c|c|c|c|c|c|c|c||}
\hline$m_{H}[\mathrm{GeV}]$ & $|X|$ & $|Y|$ & $|Z|$ & $|X Y|$ & $|X Z|$ & $|Y Z|$ & $\operatorname{Im}\left(X Y^{*}\right)$ & $\operatorname{Im}\left(X Z^{*}\right)$ \\
\hline 45 & 130 & 1.3 & 85 & 4 & 650 & 110 & 2 & 465 \\
\hline 200 & 130 & 1.9 & 340 & 8 & 12800 & 650 & 4 & 9200 \\
\hline
\end{tabular}

Table 2: The upper bounds on the MHDM parameters for a representative value of $m_{t}=140 \mathrm{GeV}$.

- Processes that involve the photon penguin diagrams depend on $X Y^{*}$. In 2HDM, the intermediate charged scalar enhances the effective $b s \gamma$ vertex. In MHDM, the intermediate charged scalar may cause larger enhancement or, when $\arg \left(X Y^{*}\right) \sim \pi$, suppress the effective $b s \gamma$ vertex. Thus, processes like $b \rightarrow s \gamma$ and $B \rightarrow X_{s} \ell^{+} \ell^{-}$may have different predictions in MHDM and in 2HDM.

\subsection{CP violation}

Within MHDM additional CP violating phases in charged scalar exchange are allowed. Those phases are absent in 2HDM. CP violation from charged scalar exchange can make only a very small contribution to $\epsilon$ (less then 4\%), and cannot be the only source of CP violation [8]. The charged scalar exchange may have a small effect on $\mathrm{CP}$ asymmetries in neutral $B$ decays, at most 0.02 shift in the measured CP asymmetries [9]. However, large contributions to the NEDM and to CP violation in top decays [71] are possible.

\section{Summary}

We have studied the charged Higgs sector of the general Multi-Higgs-Doublet Model (MHDM). Using experimental data and the requirement of perturbativity we constrained the model parameters. These bounds are summarized in Table 2 for a representative value of $m_{t}=140 \mathrm{GeV}$.

We pointed out differences between the general MHDM to the widely discussed 2HDM (Model II):

- The bound on the charged scalar mass is lower in MHDM. Thus, the charged scalar of MHDM are in the mass range accessible by LEP200.

- The bounds on $|X|$ and $|Z|$ are weaker in MHDM. Then, charged scalar exchange can saturate the experimental bound on $D-\bar{D}$ mixing, $B_{s}-\bar{B}_{s}$ can be enhanced and strong violation from lepton universality is possible.

- Large effects are possible in FCNC decays with third generation leptons. In these processes the charged scalar box diagrams are important only in MHDM.

- There are differences in the predictions for processes that involve the photon penguin diagrams, namely, $b \rightarrow s \gamma$ and $B \rightarrow X_{s} \ell^{+} \ell^{-}$. 
- In 2HDM there is no source of CP violation from the Higgs sector. In MHDM there are new $\mathrm{CP}$ violating sources but only when the charged scalars are not degenerate. Large $\mathrm{CP}$ violation effects, that arise from the CKM matrix, are possible in $D$ decays.

We conclude that MHDM may have large effects in various processes. Many of these effects do not appear in the 2HDM.

\section{Acknowledgments}

I thank Yossi Nir for his help throughout this work, Miriam Leurer and Zoltan Ligeti for useful conversations. I am grateful to Donald Finnell for providing me a computer program that enabled the calculation of $\nabla_{b}$.

\section{A Proofs of relations}

In this appendix we give proofs of relations between the coupling constants of MHDM.

\section{Relation 1}

$$
\sum_{i=2}^{n} X_{i} Y_{i}^{*}=1 \quad\left(\text { for } \Phi_{d} \neq \Phi_{u}\right)
$$

For $\Phi_{d} \neq \Phi_{u}$, unitarity of $U$ gives

$$
\sum_{i=1}^{n} U_{d i} U_{i u}^{*}=0 \Rightarrow \sum_{i=2}^{n} U_{d i} U_{i u}^{*}=-U_{d 1} U_{1 u}^{*} \Rightarrow \frac{\sum_{i=2}^{n} U_{d i} U_{i u}^{*}}{U_{d 1} U_{1 u}^{*}}=-1 .
$$

Using the definition of $X_{i}$ and $Y_{i}$ (eq. (6)) we get eq. (76). The proof of the other two relations in eq. (7) is obtained in a similar way.

\section{Relation 2}

$$
\sum_{i=2}^{n}\left|X_{i}\right|^{2}=\frac{v^{2}}{v_{d}^{2}}-1
$$

Using eq. (6), eq. (5) and the unitarity of $U$ we get

$$
\sum_{i=2}^{n}\left|X_{i}\right|^{2}=\frac{\sum_{i=2}^{n}\left|U_{d i}\right|^{2}}{\left|U_{d 1}\right|^{2}}=\frac{\sum_{i=1}^{n}\left|U_{d i}\right|^{2}}{\left|U_{d 1}\right|^{2}}-1=\frac{1}{\left|U_{d 1}\right|^{2}}-1=\frac{v^{2}}{v_{d}^{2}}-1 .
$$

The proof of the other two relations in eq. (8) is obtained in a similar way.

Relation $3 X_{i}, Y_{j}$ and $Z_{k}(i, j, k \in 2 . . n)$ cannot be simultaneously large (more than $\sqrt{n-1}$ ), unless there is at least one Higgs doublet that does not couple to fermions. 
The proof is given for a general case where there are $m$ types of massive fermions and $n$ Higgs doublets. We denote the coupling of each fermion type by $X^{k}$, where $k$ is the fermion type. We demand

$$
\left|X_{i}^{k}\right|>a
$$

Using eq. (8) we get

$$
a^{2}<\left|X_{i}^{k}\right|^{2} \leq \sum_{i=2}^{n}\left|X_{i}^{k}\right|^{2}=\frac{v^{2}}{v_{k}^{2}}-1 \Rightarrow v^{2}>\left(a^{2}+1\right) v_{k}^{2}
$$

Summing over all fermions types we get

$$
m v^{2}>\left(a^{2}+1\right) \sum_{k=1}^{m} v_{k}^{2} .
$$

We have to distinguish between two cases. First, $m=n$ and each fermion type couples exactly to one Higgs doublets. Then eq. (82) with eq. (5) lead to

$$
n v^{2}>\left(a^{2}+1\right) v^{2} \Rightarrow a<\sqrt{n-1}
$$

On the other hand, if there is at least one Higgs doublet that does not couple to fermions, $a$ is not bounded.

\section{B Formulae}

In this appendix we give the expressions of various functions that play a role in our study.

\section{B.1 Neutral meson mixing}

All the integrals that we have to calculate are of the form

$$
J_{n}(a, b, c, d)=\int_{0}^{\infty} \frac{x^{n} d x}{(x+a)(x+b)(x+c)(x+d)},
$$

for $n=0,1,2$. We get

$$
\begin{aligned}
J_{n}= & \frac{a^{n} \ln (a)}{(a-b)(a-c)(a-d)}+\frac{b^{n} \ln (b)}{(b-a)(b-c)(b-d)}+ \\
& \frac{c^{n} \ln (c)}{(c-a)(c-b)(c-d)}+\frac{d^{n} \ln (d)}{(d-a)(d-b)(d-c)} .
\end{aligned}
$$

The integrals in the text are

$$
\begin{aligned}
I_{1}\left(y_{t}\right) & =J_{2}\left(1,1, y_{t}, y_{t}\right)=\frac{1+y_{t}}{\left(1-y_{t}\right)^{2}}+\frac{2 y_{t} \ln \left(y_{t}\right)}{\left(1-y_{t}\right)^{3}} \\
I_{2}\left(y_{t}\right) & =J_{1}\left(1,1, y_{t}, y_{t}\right)=\frac{2}{\left(1-y_{t}\right)^{2}}+\frac{\left(1+y_{t}\right) \ln \left(y_{t}\right)}{\left(1-y_{t}\right)^{3}}, \\
I_{3}\left(x_{t}, x_{H}\right) & =J_{2}\left(1, x_{H}, x_{t}, x_{t}\right)=
\end{aligned}
$$




$$
\begin{aligned}
& \frac{x_{t}}{\left(x_{t}-x_{H}\right)\left(1-x_{t}\right)}+\frac{x_{H}^{2} \ln \left(x_{H}\right)}{\left(1-x_{H}\right)\left(x_{t}-x_{H}\right)^{2}}+\frac{x_{t}\left(x_{t}+x_{t} x_{H}-2 x_{H}\right) \ln \left(x_{t}\right)}{\left(1-x_{t}\right)^{2}\left(x_{t}-x_{H}\right)^{2}}, \\
I_{4}\left(x_{t}, x_{H}\right)= & J_{1}\left(1, x_{H}, x_{t}, x_{t}\right)= \\
& \frac{1}{\left(x_{t}-x_{H}\right)\left(1-x_{t}\right)}+\frac{x_{H} \ln \left(x_{H}\right)}{\left(1-x_{H}\right)\left(x_{t}-x_{H}\right)^{2}}+\frac{\left(x_{t}^{2}-x_{H}\right) \ln \left(x_{t}\right)}{\left(1-x_{t}\right)^{2}\left(x_{t}-x_{H}\right)^{2}}, \\
I_{5}\left(y_{b}, y_{s}\right)= & J_{2}\left(1,1, y_{s}, y_{b}\right) .
\end{aligned}
$$

The SM integral can also be obtained from the general integral $J$. However, we keep the conventional notation [22]

$$
\begin{aligned}
I_{0}(y) & =1-\frac{3 y(1+y)}{4(1-y)^{2}}\left[1+\frac{2 y \ln (y)}{1-y^{2}}\right], \\
I_{6}(x, y) & =\ln (y / x)-\frac{3 y}{4(1-y)}\left[1+\frac{y \ln (y)}{1-y}\right] .
\end{aligned}
$$

For the calculations of the matrix elements we use the Vacuum Insertion Approximation

$$
\begin{aligned}
V_{L L} & =\left\langle\bar{B}\left|\left[\bar{d} \gamma^{\nu}\left(1-\gamma_{5}\right) b\right]\left[\bar{d} \gamma_{\nu}\left(1-\gamma_{5}\right) b\right]\right| B\right\rangle=\frac{1}{3} f_{B}^{2} m_{B} \\
S_{L L} & =\left\langle\bar{B}\left|\left[\bar{d}\left(1-\gamma_{5}\right) b\right]\left[\bar{d}\left(1-\gamma_{5}\right) b\right]\right| B\right\rangle=-\frac{5}{6}\left(\frac{m_{B}}{m_{b}+m_{d}}\right)^{2} f_{B}^{2} m_{B} .
\end{aligned}
$$

B.2 $Z \rightarrow b \bar{b}$

with

$$
C=\frac{\alpha v_{L}}{2 \pi \sin ^{2} \theta_{W}\left(v_{L}^{2}+v_{R}^{2}\right)}
$$

$$
v_{L}=-\frac{1}{2}+\frac{1}{3} \sin ^{2} \theta_{W}, \quad v_{R}=\frac{1}{3} \sin ^{2} \theta_{W} .
$$

B.3 $b \rightarrow s \gamma$

$$
\begin{aligned}
G_{W}(x) & =\frac{x}{12(1-x)^{4}}\left[\left(7-5 x-8 x^{2}\right)(1-x)+6 x(2-3 x) \ln (x)\right] \\
G_{H}(x) & =\frac{-x}{6(1-x)^{3}}[(3-5 x)(1-x)+2(2-3 x) \ln (x)] \\
F_{p s}(x) & =1-8 x+8 x^{3}-x^{4}-12 x^{2} \ln (x) .
\end{aligned}
$$

B.4 $K^{+} \rightarrow \pi^{+} \nu \bar{\nu}$

$$
\begin{aligned}
D_{S M}(x, y)= & \frac{1}{8}\left\{\frac{x y}{y-x}\left(\frac{4-y}{1-y}\right)^{2} \ln (y)-2 x-\left(1-\frac{3}{1-y}\right) \frac{3 x}{1-x}\right. \\
& \left.-\left[\frac{x}{y-x}\left(\frac{4-x}{1-x}\right)^{2}+1+\frac{3}{(1-x)^{2}}\right] x \ln (x)\right\}, \\
D_{Z H}(x, y)= & \frac{x y}{4} \frac{1-y+\ln (y)}{(1-y)^{2}}, \\
I_{7}\left(x_{t}, x_{\ell}, x_{H}\right)= & J_{2}\left(1, x_{t}, x_{\ell}, x_{H}\right), \\
I_{8}\left(x_{t}, x_{\ell}, x_{H}\right)= & J_{1}\left(1, x_{t}, x_{\ell}, x_{H}\right) .
\end{aligned}
$$




\section{B.5 $B \rightarrow X \tau \nu_{\tau}$}

The phase space factors for three body decay with only one massless anti-particle in the final state are 72,53

$$
\begin{aligned}
\tilde{F}_{p s}(x, y) & =12 \int_{(x+y)^{2}}^{1} \frac{d s}{s}\left(s-x^{2}-y^{2}\right)(1-s)^{2} g\left(s, x^{2}, y^{2}\right), \\
\tilde{F}_{p s}^{i n t}(x, y) & =6 \int_{x^{2}}^{(1-y)^{2}} \frac{d s}{s}\left(s-x^{2}\right)^{2} g\left(1, s, y^{2}\right),
\end{aligned}
$$

with

$$
g(a, b, c)=\sqrt{a^{2}+b^{2}+c^{2}-2(a b+a c+b c)} .
$$

We do not give the analytic expressions of these functions. $\tilde{F}_{p s}$ can be found in [72, while we use a computer program to calculate $\tilde{F}_{p s}^{i n t}$.

\section{B.6 $e-\mu$ universality in $\tau$ decays}

The phase space function for the interference term can be found in [57]

$$
F_{p s}^{i n t}(x)=1+9 x-9 x^{2}-x^{3}+6 x(1+x) \ln (x) .
$$

B.7 $\quad B \rightarrow \ell^{+} \ell^{-}$

$$
\begin{aligned}
G^{S M}(x) & =\frac{1}{2}\left(x+\frac{3 x}{1-x}+\frac{3 x^{2} \ln (x)}{(1-x)^{2}}\right) \\
J\left(y_{t}\right) & =\frac{1-y+\ln (y)}{2(1-y)^{2}}, \\
I_{9}\left(y_{t}\right) & =J_{2}\left(1,1, y_{t}, 0\right)=\frac{1-y_{t}-y_{t} \ln \left(y_{t}\right)}{\left(1-y_{t}\right)^{2}}, \\
I_{10}\left(x_{t}, x_{H}\right) & =J_{2}\left(1, x_{t}, x_{H}, 0\right)=\frac{x_{t} \ln \left(x_{t}\right)}{\left(1-x_{t}\right)\left(x_{H}-x_{t}\right)}+\frac{x_{H} \ln \left(x_{H}\right)}{\left(1-x_{H}\right)\left(x_{t}-x_{H}\right)} .
\end{aligned}
$$

\section{References}

[1] J.F. Gunion, H.E. Haber, G.L. Kane and S. Dawson, The Higgs Hunter's Guide (Addison-Wesley Publishing Company, Reading, MA, 1990) and references therein.

[2] K. Hikasa et al. (Particle Data Group), Phys. Rev. D45 (1992) S1.

[3] S.L. Glashow and S. Weinberg, Phys. Rev. D15 (1977) 1958.

[4] C. Albright, J. Smith and S.-H.H. Tye, Phys. Rev. D21 (1980) 711.

[5] G.C. Branco, A.J. Buras and J.-M. Gerard, Nucl. Phys. B259 (1985) 306.

[6] S. Weinberg, Phys. Rev. Lett. 37 (1976) 657. 
[7] G.C. Branco, Phys. Rev. lett. 44 (1980) 504.

[8] P. Krawczyk and S. Pokorski, Nucl. Phys. B364 (1991) 10.

[9] Y. Grossman and Y. Nir, Phys. Lett. B313 (1993) 126.

[10] J. Gasser and H.Leutwyler, Phys. Rep. 87 (1982) 77.

[11] D. Amidei, CDF Collaboration, talk given in the fifth international symposium on heavy flavor physics, Montreal, Canada (1993).

[12] A. Blondel and C.Verzegnassi, Phys. Lett B311 (1993) 346.

[13] V. Barger and R.J.N. Phillips, Phys. Rev. D41 (1990) 884.

[14] A. Denner, R.J. Guth, J.H. Kühn, Phys. Lett. B240 (1990) 438.

[15] Y. Nir, Lectures presented in TASI-91, SLAC-PUB-5676 (1991) and references therein.

[16] Y. Nir and U. Sarid, Phys. Rev. D47 (1993) 2818 and references therein.

[17] J. Bartelt et al., CLEO Collaboration, Phys. Rev. lett. 71 (1993) 4111.

[18] A.J. Buras, P. Krawczyk, M.E. Lautenbacher and C. Salazar, Nucl. Phys. B337 (1990) 284.

[19] A. Sopczak, CERN-PPE/93-86.

[20] V. Barger, J.L. Hewett and R.J.N. Phillips, Phys. Rev. D41 (1990) 3421.

[21] P.J. Franzini, Phys. Rep. 173 (1989) 1.

[22] Y. Nir, Lectures presented in the 20th SLAC Summer Institute, SLAC-PUB-5874 (1992) and references therein.

[23] C.T. Sachrajda, Nucl. Phys. B. Proc. Supp. 30 (1992) 20.

[24] A.J. Buras, M. Jamin and P.H. Weisz, Nucl. Phys. B347 (1990) 491.

[25] G. Buchalla, A.J. Buras, M.K. Harlander, M.E. Lautenbacher and C. Salazar, Nucl. Phys. B355 (1991) 305.

[26] L.F. Abbott, P. Sikivie and M.B. Wise, Phys. Rev. D21 (1980) 1393.

[27] G.G. Athanasiu, P.J. Franzini and F.J. Gilman, Phys. Rev. D32 (1985) 3010.

[28] M. Neubert, Phys. Rev. D45 (1992) 2451.

[29] G.G. Athanasiu and F.J. Gilman, Phys. Lett. B153 (1985) 274.

[30] H.-Y. Cheng, Int. J. Mod. Phys. A7 (1992) 1059. 
[31] Y. Dupont and T.N. Pham, Phys. Rev. D28 (1983) 2169;

H.Y. Cheng, Phys. Rev. D34 (1986) 1397;

J.S. Hagelin, Phys. Lett. B117 (1982) 441;

H.Y. Cheng, Phys. Rev. D42 (1990) 2329.

[32] I.I. Bigi and A.I. Sanda, Phys. Lett. B171 (1986) 320;

I.I. Bigi, UND-HEP-89-BIG01 (1989).

[33] L. Wolfenstein, Phys. Lett. B164 (1985) 170;

J.F. Donoghue et al., Phys. Rev. D33 (1986) 179.

[34] H. Georgi, Phys. Lett. B297 (1992) 353;

T. Ohl, G. Ricciardi and E.H. Simmons, Nucl. Phys B403 (1993) 605.

[35] G. Altarelli, R. Barbieri and F. Caravaglios, CERN-TH.6859/93.

[36] G.T. Park, CTP-TAMU-69/93.

[37] M. Boulware and D. Finnell, Phys. Rev. D44 (1991) 2054.

[38] A. Denner, R.J. Guth, W. Hollik and J.H. Kühn, Z. Phys. C51 (1991) 695.

[39] E. Thorndike, CLEO Collaboration, talk given in the meeting of the American Physical Society, Washington D.C. (1993).

[40] A.J. Buras, M. Misiak, M. Münz and S. Pokorski, MPI-Ph/93-77 (1993).

[41] T. Inami and C.S. Lim, Prog. Theor. Phys. 65 (1981) 297; (E) 65 (1981) 1772.

[42] W.-S. Hou and R.S. Willey, Phys. Lett. B202 (1988) 591; Nucl. Phys. B326 (1989) 54.

[43] T.G. Rizzo, Phys. Rev. D38 (1988) 820.

[44] B. Grinstein, R. Springer and M. Wise, Nucl. Phys. B339 (1990) 269.

[45] E. Ma and A. Parmudita, Phys. Rev D24 (1981) 1410.

[46] M.S. Atiya et al., E787 collaboration, Phys. Rev. Lett. 70 (1993) 2521.

[47] I.I. Bigi and F. Gabbiani, Nucl. Phys. B367 (1991) 3.

[48] C.Q. Geng and J.N. Ng, Phys. Rev. D38 (1988) 2858; (E) 41 (1990) 1715.

[49] G. Buchalla and A.J. Buras, Nucl. Phys B398 (1993) 285.

[50] D. Buskulic et al., ALEPH collaboration, Phys. Lett. B298 (1993) 479;

A. Putzer, ALEPH Collaboration, talk given in the fifth international symposium on heavy flavor physics, Montreal, Canada (1993). 
[51] A.F. Falk, Z. Ligeti, M. Neubert and Y.Nir, WIS-93/117/Dec-PH.

[52] P. Krawczyk and S. Pokorski, Phys. Rev. Lett. 60 (1988) 182.

[53] J. Kalinowski, Phys. Lett. B245 (1990) 201;

B. Grzadkowski and W.-S. Hou, Phys. Lett. B272 (1991) 383.

[54] H. Isidori, Phys. Lett. B298 (1993) 409.

[55] A.J. Weinstein and R. Stroynowski, CALT-68-1853 (1993).

[56] H.E. Haber, G.L. Kane and T. Sterling, Nucl. Phys. B161 (1979) 493.

[57] W. Holik and T. Sack, Phys. Lett. B284 (1992) 427.

[58] M. Roney, talk given in the fifth international symposium on heavy flavor physics, Montreal, Canada (1993).

[59] G. Beall and N.G. Deshpande, Phys. Lett. B132 (1983) 427.

[60] D.A. Dicus, Phys. Rev. D41 (1990) 999.

[61] D. Chang, W.Y. Keung, C.S. Li and T.C. Yuan, Phys Lett. B241 (1990) 589.

[62] G. Buchalla and A.J. Buras, Nucl. Phys B400 (1993) 225.

[63] J.L. Hewett, S. Nandi and T.G. Rizzo, Phys. Rev. D39 (1989) 250.

[64] X.-G. He, T.D. Nguyen and R.R. Volkas, Phys. Rev. D38 (1988) 814.

[65] W. Skiba and J. Kalinowski, Nucl. Phys. B404 (1993) 3.

[66] N.G. Deshpande, K. Panose and J. Trampetic, Phys. Lett. B308 (1993) 322.

[67] D. Cinabro, CLEO Collaboration, talk given in the Fermilab Meeting DPF 92 (1992).

[68] W.-S. Hou, Phys. Rev. D48 (1993) 2342.

[69] J.L. Hewett, Phys. Rev. Lett. 70 (1993) 1045;

V. Barger, M.S. Berger and R.J.N. Phillips, Phys. Rev. Lett. 70 (1993) 1368.

[70] A. Pich, CERN-TH.7066/93.

[71] D. Atwood, G. Eilam, A. Sony, R.R. Mendel and R. Migneron, Phys. Rev. Lett. 70 (1993) 1364.

[72] J.L. Cortes, X.Y. Pham and A. Tounsi, Phys. Rev. D25 (1982) 188. 
This figure "fig1-1.png" is available in "png" format from: http://arxiv.org/ps/hep-ph/9401311v1 
This figure "fig2-1.png" is available in "png" format from: http://arxiv.org/ps/hep-ph/9401311v1 
This figure "fig1-2.png" is available in "png" format from: http://arxiv.org/ps/hep-ph/9401311v1 
This figure "fig2-2.png" is available in "png" format from: http://arxiv.org/ps/hep-ph/9401311v1 
This figure "fig1-3.png" is available in "png" format from: http://arxiv.org/ps/hep-ph/9401311v1 
This figure "fig2-3.png" is available in "png" format from: http://arxiv.org/ps/hep-ph/9401311v1 